\title{
BMJ Open Treatment of postoperative delirium with continuous theta burst stimulation: study protocol for a randomised controlled trial
}

\author{
Xiaoyi Wei, ${ }^{1,2}$ Meijuan Wang, ${ }^{1}$ Xin Ma, ${ }^{1,2}$ Tianyi Tang, ${ }^{1,2}$ Jingqing Shi, ${ }^{1}$ Di Zhao, ${ }^{3}$ \\ Tifei Yuan, ${ }^{3}$ Zhongcong Xie, ${ }^{4}$ Yuan Shen (i) ${ }^{1,2}$
}

To cite: Wei X, Wang M, Ma X, et al. Treatment of postoperative delirium with continuous theta burst stimulation: study protocol for a randomised controlled trial. BMJ Open 2021;11:e048093. doi:10.1136/ bmjopen-2020-048093

- Prepublication history and additional supplemental material for this paper are available online. To view these files, please visit the journal online (http://dx.doi.org/10.1136/ bmjopen-2020-048093).

XW and MW contributed equally.

Received 16 December 2020 Accepted 04 July 2021

Check for updates

(c) Author(s) (or their employer(s)) 2021. Re-use permitted under CC BY-NC. No commercial re-use. See rights and permissions. Published by BMJ.

For numbered affiliations see end of article.

\section{Correspondence to}

Dr Zhongcong Xie;

zxie@mgh.harvard.edu and

Dr Yuan Shen;

kmshy@tongji.edu.cn

\section{ABSTRACT}

Introduction Postoperative delirium is one of the most common postoperative complications among elderly patients (65 years old or older). However, there are no effective treatments for this condition. Recent research suggests that continuous theta burst stimulation (cTBS), a non-invasive brain stimulation, can reduce pain level, improve cognitive function and affective symptoms in multiple diseases or dysfunctions, including anxiety disorders, major depressive disorder, sleep disorders and pain. But the potential benefits of cTBS in reducing postoperative delirium have not been investigated. Therefore, we propose determining whether cTBS can prevent and/or treat postoperative delirium in senior patients.

Methods and analysis The study will be a double-blind, randomised controlled trial. Participants (65 years old or older) undergoing scheduled orthopaedic surgery ( $\geq 2$ hours, general anaesthesia) will be randomised to receive either cTBS or sham stimulation with a focal figure-ofeight coil over the right dorsolateral prefrontal cortex at $80 \%$ of the resting motor threshold. Every patient will receive $2-3$ sets of stimulations during postoperative days (40 s per session, 3 sessions per set, 1 set per day). Participants will be assessed twice daily by a research assistant blinded to allocation. The primary outcome will be the incidence of postoperative delirium measured by the Confusion Assessment Method on postoperative days 1,2 and 3 . The secondary outcomes will be the severity and duration of postoperative delirium, cognitive function, pain, sleep quality, activities of daily living, length of hospital stay, discharge-to-facility or home, and rate of complication and mortality during the hospital stay.

Ethics and dissemination Ethical approval has been obtained from the ethics committee of Shanghai 10th People's Hospital. The principal investigator will submit a research progress report to the ethics committee regularly. All participants will provide written informed consent. Study results will be published in a peer-reviewed journal. Trial registration number NCT04661904.

\section{INTRODUCTION}

Postoperative delirium is one of the most common postoperative complications in elderly patients ${ }^{1}$ and is associated with
Strengths and limitations of this study

- We have developed a system to investigate postoperative delirium in our institute, including standardised tools, reliable assessments and a collaborative team comprising of surgeons, anaesthesiologists and psychiatrists.

- We have established methods to determine the incidence of postoperative delirium (primary outcome) from our previous studies.

- We will use secondary outcomes to further illustrate the effects of theta burst stimulation, including severity and duration of postoperative delirium, cognitive function, pain, sleep quality, activities of daily living, length of hospital stay, discharge-to-facility or home, rate of complication and mortality during the hospital stay.

- The proposed research will involve collaborators who are experts in brain stimulation research, which will enhance the safety and efficacy of this intervention study.

- The proposed investigation is not a multicentre study.

short-term and long-term cognitive impairment ${ }^{2-4}$ and dementia. ${ }^{5}$ Delirium occurs in $25 \%-50 \%$ of elderly surgical patients and is associated with increased risk of developing Alzheimer's disease and related dementias (ADRD), greater incidence of postoperative complications, higher mortality, prolonged hospital stays and higher discharge rates to nursing homes. ${ }^{16-9}$ Our previous studies consistently showed that the incidence of postoperative delirium in patients with scheduled hip surgery was about 25\%. ${ }^{10}{ }^{11}$ Patients with dementia are 2.5-4.7 times more likely to develop delirium, and patients with delirium face a 12.5-fold increased incidence of newly diagnosed ADRD. ${ }^{6}{ }^{12-14}$ US healthcare costs attributable to delirium exceed US $\$ 182$ billion per year. ${ }^{15} 16$ The Hospital Elder Life Programme ${ }^{17}$ works with the hospital nursing 
staff to address the full scope of geriatric issues and iatrogenic complications contributing to cognitive and functional decline during the hospitalisation. However, there are currently few effective treatments for postoperative delirium. Therefore, it is essential to identify effective interventions to treat or prevent postoperative delirium.

Recently, brain stimulation was demonstrated to improve cognitive function in patients with mild cognitive impairment. ${ }^{18}$ Transcranial magnetic stimulation (TMS) is a commonly used brain stimulation technique that selectively modulates neuronal activity by generating electrical currents through an electromagnetic coil. TMS can enhance neuroplasticity, the ability of the brain to form new nerve connections. ${ }^{19}$ Furthermore, neural activity can be modulated using different repetitive TMS (rTMS) patterns. ${ }^{20}{ }^{21}$ Previous studies with low-frequency rTMS applied over the right dorsolateral prefrontal cortex (DLPFC) have shown a significant increase in the connectivity and reorganisation of the brain network and improvement of cognitive performance. ${ }^{22} 23$

Compared with low-frequency rTMS, continuous theta burst stimulation (cTBS) requires a shorter application time to be effective, making cTBS safer and more acceptable for patients. ${ }^{24}$ cTBS is a non-invasive brain stimulation, and every session of cTBS performed the figure-of-eight coil in this study lasts $40 \mathrm{~s}$ (600 pulses). The cTBS efficacy on the cortex lasts for $50 \mathrm{~min}$, and the variation in effects is more significant between different stimulation types within $30 \mathrm{~min}$ after stimulation. Indeed, cTBS over the right DLPFC improves decision making by reducing impulsive choices and favouring delayed large rewards instead of immediate small rewards..$^{25} 26$ cTBS can also enhance other executive functions. ${ }^{25}{ }^{27}$ Furthermore, cTBS has analgesic effects and can improve sleep quality. ${ }^{28}{ }^{29}$ However, no clinical studies have determined whether cTBS can prevent or treat postoperative delirium in patients.

Therefore, we plan to conduct a randomised controlled double-blind study in participants (65 years old or older) undergoing elective orthopaedic surgeries to determine whether cTBS can reduce the incidence and severity of postoperative delirium in this patient population. We hypothesise that postoperative cTBS intervention will reduce the incidence of postoperative delirium in senior patients.

\section{METHODS AND ANALYSIS \\ Study design and setting}

This study will be a single-centre, randomised, doubleblind, sham-controlled, parallel-group trial. We will use Confusion Assessment Method (CAM) ${ }^{1}$ to determine the incidence of postoperative delirium in participants. We will use the CAM-based Scoring System for Delirium Severity $(\mathrm{CAM}-\mathrm{S})^{30}$ to examine postoperative delirium severity. The average duration of postoperative delirium will be calculated by dividing the total days that postoperative delirium occurs by the number of participants. The

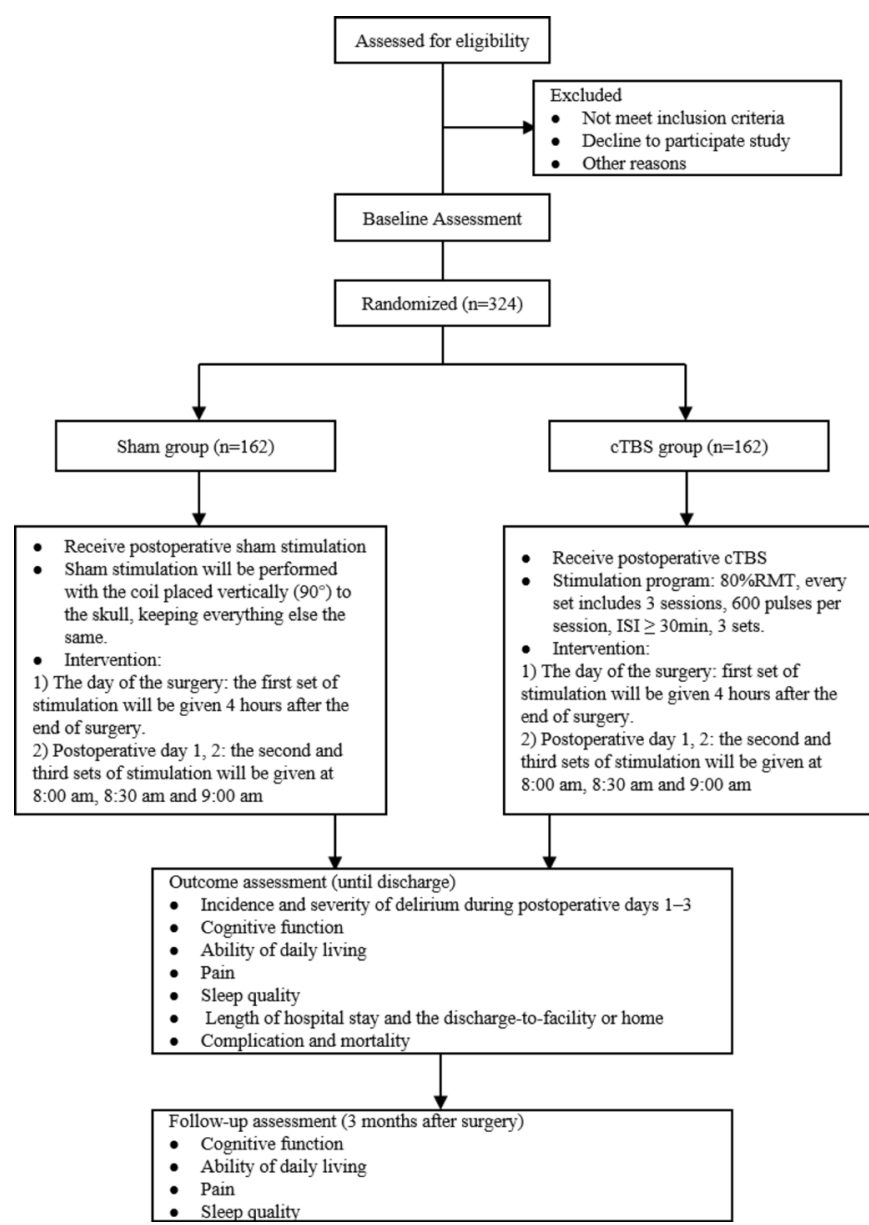

Figure 1 Study flow chart. The present study is a randomised controlled trial. The enrolled participants will be randomised equally into two groups. After surgery, each participant will be allocated to receive sham or active stimulation. the incidence and severity of postoperative delirium following cTBS or sham stimulation will be recorded and analysed to determine potential differences between the two cohorts. cTBS, continuous theta burst stimulation; ISI, intersession interval; RMT, resting motor threshold.

study will consist of two arms: a cTBS cohort and a sham stimulation cohort. All recruitment and intervention in the participants will be performed in Shanghai 10th People's Hospital (Shanghai, China). The overall study design is illustrated in figure 1 , and the study schedule is presented in figure 2.

\section{Sample size and statistical power}

We hypothesise that cTBS will decrease the incidence of postoperative delirium compared with sham stimulation. We will include the participants with orthopaedic surgery because our previous studies have established a system to investigate postoperative delirium in the participants with orthopaedic surgery. Moreover, the participants with orthopaedic surgery have fewer contraindications (eg, cerebral haemorrhage, massive cerebral infarction, cardiac pacemaker and other implants in the body) of rTMS. Our previous studies demonstrated that the 


\begin{tabular}{|l|c|c|c|c|c|c|c|c|}
\hline \multicolumn{1}{|c|}{ Content } & Screning & Baseline & \multicolumn{5}{|c|}{ Postoperative hospitalization } \\
\hline \multicolumn{1}{|c|}{ Time point } & Preoperative & Preoperative & Surgery day & Dayl & Day2 & Day 3 & Discharge & Follow-up \\
\hline Demographic data & $\times$ & & & & & & & \\
\hline Signaturc of ICF & $\times$ & & & & & & & \\
\hline $\begin{array}{l}\text { Inclusion and } \\
\text { Exclusion criteria }\end{array}$ & $\times$ & & & & & & & \\
\hline Intervention & & & $\times$ & $\times$ & $\times$ & & & \\
\hline MMSE & & $\times$ & & & & & & $\times$ \\
\hline DSI Daily & & $\times$ & & $\times$ & $\times$ & $\times$ & $\times$ & $\times$ \\
\hline CAM, CAM-S & & $\times$ & & $\times$ & $\times$ & $\times$ & & \\
\hline ADL & & $\times$ & & & & & $\times$ & $\times$ \\
\hline VAS & & $\times$ & & $\times$ & $\times$ & $\times$ & $\times$ & $\times$ \\
\hline Sleep quality & & $\times$ & & $\times$ & $\times$ & $\times$ & $\times$ & $\times$ \\
\hline
\end{tabular}

Figure 2 Study schedule. Description of the study protocol. $A D L$, activities of daily living; CAM, Confusion Assessment Method; CAM-S, Confusion Assessment Method basedScoring System for Delirium Severity; DSI, Delirium Symptom Interview; ICF, Informed Consent Form; MMSE, Mini-Mental State Examination; VAS, Visual Analogue Scale.

incidence of postoperative delirium in elective hip surgery is about $25 \% .{ }^{1011}$ However, there have been no studies to determine the effects of cTBS on postoperative delirium incidence. Thus, in this study, we investigated the effects of low-frequency rTMS on a major depressive disorder to calculate the sample size. Given that rTMS showed over a $50 \%$ reduction in the incidence of major depression, ${ }^{31} 32$ we estimated that cTBS would reduce the postoperative delirium incidence up to $50 \%$ (from $25.6 \%{ }^{10}$ to $12.8 \%$ ) in this pilot study. We, therefore, performed power analysis based on a two-sided chi-square test and calculated that 145 participants in each group would be necessary to draw such a difference with a significance level of 0.05 and a power of the test of 0.8 . Considering the drop-out rate of $10 \%$ in our previous study, ${ }^{33}$ the sample size will be 162 participants in each group. If we observe more drop-out from the study, we will recruit more participants to achieve a final number of 145 in each group.

\section{Study population}

\section{Inclusion criteria}

Participants will be screened for inclusion if they meet the following criteria:

- Age $\geq 65$ years old.

- Having a scheduled surgery ( $\geq 2$ hours, general anaesthesia) (eg, proximal femoral nail, hip replacement, open reduction and internal fixation of hip and lumbar spine). The emergent surgeries due to hip fracture are not included.

- Being able to complete cognitive assessments and CAM.

- Having normal cognitive function at the time of enrolment with Mini-Mental State Examination (MMSE) scores equal to or above 24.

- Chinese Mandarin as their native language.

- Being willing to participate and being competent to provide informed consent.
Exclusion criteria

Participants who meet any of the following criteria will be excluded:

- Having delirium, assessed by CAM, before surgery.

- Having a brain tumour, stroke or mental disorders (eg, major depressive disorder or dementia).

- Having contraindications of cTBS (eg, head trauma, history of epilepsy or metal implants in the head).

- Participating in other clinical studies at the time of screening.

- Having postoperative complications such as pulmonary infection, pulmonary embolism and stroke.

\section{Stopping criteria for cTBS}

Participants will stop receiving stimulation if any of the following occurs:

- Serious adverse events (eg, epilepsy, syncope, severe headache) due to the application of cTBS.

- Refusal to continue to accept stimulation.

- Contraindications such as cerebral infarction due to the surgery.

- Temporary surgeries such as installation of the pacemaker during the hospitalisation.

\section{Termination criteria}

A data safety monitoring board (DSMB) will supervise the study to review safety and efficacy of data periodically. This study will be terminated if any of the following occurs:

- Unexpected or frequent serious adverse events, including epilepsy, syncope, severe headache, due to the application of cTBS.

- Serious errors, including ethics problems, low data quality or randomisation error found in the study protocol.

- Low positive rate or futility result reported in the interim analysis.

- Failure to maintain a double-blind status in the trial.

If the study is terminated early, a written report will be submitted to the medical instrument clinical trial management department in the Shanghai 10th People's Hospital within 5 days. The trial cannot be resumed without the ethics committee's approval in the Shanghai 10th People's Hospital.

\section{Purpose of the study}

Primary outcome

Postoperative delirium incidence.

Postoperative delirium will be defined according to $\mathrm{CAM},{ }^{1}$ which includes four entries: acute onset and fluctuating course, inattention, disorganised thinking and altered level of consciousness. To diagnose delirium, both the first and second criteria have to be present and the third and/or fourth criteria. Therefore, every participant will be assessed twice daily after surgery, with the first assessment at 9:00-10:00 hours. The second assessment will be at 16:00-17:00 hours, about 7 hours after the first assessment on postoperative days 1, 2 and 3 . 


\section{Secondary outcomes}

\section{Postoperative delirium severity}

The severity of delirium will be assessed by the CAMS. ${ }^{30}$ CAM-S includes 10 items: acute onset or symptom fluctuation, inattention, disorganised thinking, altered level of consciousness, disorientation, memory impairment, perceptual disturbances, psychomotor agitation, psychomotor retardation and sleep-wake cycle disturbance. Each item is scored as absent (0), mild (1) or marked (2), except acute onset or fluctuation, which is scored as absent (0) or present (1). The sum of CAM-S ranges from 0 (none) to 19 (most severe). The Delirium Symptom Interview (DSI) Daily ${ }^{34}$ will also be used to determine CAM-S grades. The incidence of postoperative delirium will also be referred to the records by nurses on duty.

\section{Postoperative delirium duration}

Postoperative delirium duration will be determined by dividing the total days when postoperative delirium occurs by the total number of participants.

\section{Cognitive function related to delirium}

We will use the DSI Daily ${ }^{34}$ to assess the cognitive function associated with delirium. The DSI Daily includes seven domains: disorientation, disturbance of consciousness, disruption of sleep/wake cycle, perceptual disturbance, the incoherence of speech, change in psychomotor activity, and fluctuating behaviour. DSI Daily will also be used to grade the CAM-S.

\section{Pain}

Pain will be assessed using the Visual Analogue Scale (VAS). Participants will be scored according to the severity of their pain, from no pain (0) to most painful (10).

\section{Sleep quality}

Sleep quality will be assessed using the Pittsburgh Sleep Quality Index (PSQI). The PSQI contains 19 self-rated questions combined to form seven component scores, including subjective sleep quality, sleep latency, sleep duration, habitual sleep efficiency, sleep disturbances, use of sleeping medication and daytime dysfunction. Each item is weighted on a $0-3$ interval scale. Then, the global PSQI score is calculated by totalling the seven component scores, providing an overall score ranging from 0 to 21, where lower scores indicate better sleep quality.

\section{Activities of daily living}

Activities of daily living (ADL) will be assessed using the Chinese version of the ADL scale, including a Physical Self-Maintenance Scale and an Instrumental ADL at baseline (before the surgery) and follow-ups. There are a total of 14 items in the ADL. For each item, scoring is determined by a 4-point coding system: 1 = 'can do it myself,' $2=$ 'have some difficulty but can still do it by myself,' $3=$ 'need help to do it,' and $4=$ 'cannot do it at all.' The total ADL score ranges from 14 to 56 points, and higher ADL scores indicate lower ADL. Participants with ADL scores of more than 22 points (cut-off score) will be defined as having impairment in ADL.

\section{Length of hospital stay and discharge-to-facility or home}

Length of hospital stay and discharge-to-facility or home will also be taken into consideration.

Rate of complication and mortality during the hospital stay or up to 7 days after anaesthesia/surgery, whichever is longer

Every participant complication will be recorded to calculate the complication rate.

\section{Randomisation and blinding}

Informed consent will be obtained from the participants before surgery. A researcher who is independent of the study will be responsible for randomisation and allocation at the beginning of the study. Each participant will be randomly assigned to the cTBS stimulation group or sham stimulation group according to an allocated randomisation number (in a 1:1 ratio using Excel for Windows). The randomised numbers will be sealed in sequentially numbered envelopes and stored at the study site. The participants will be recruited after being admitted to the ward. The clinical research coordinator or graduate student who administers the cTBS will open the sealed envelope containing the allocation and randomisation number 1 day before the intervention. Another research assistant who is blinded to the randomisation and allocations will perform the assessments in the participants. The assessment and intervention will be performed in the same location as the participants. The allocations of participants should only be unblended in exceptional circumstances under which the actual treatment is essential for managing participants.

\section{Description of the study intervention}

The minimum number of stimulations that will be performed in the participants are two sets on the day of surgery and postoperative day 1 , respectively. The maximum number of stimulations are three sets on the day of surgery and postoperative days 1 and 2. Only the participants who finish the minimum stimulations or more will be included in the per-protocol set. Data from participants who fail to accept the minimum stimulations for any reason will be used for intention-to-treat analysis.

\section{CTBS group}

cTBS will be performed using a commercially available magnetic stimulator (YRD CCY-I, Wuhan Yiruide Medical Equipment, Wuhan, China) equipped with a focal figure-of-eight coil. Every session of cTBS consists of triplets of $50 \mathrm{~Hz}$ pulses delivered at $5 \mathrm{~Hz}$ for $40 \mathrm{~s}$ for 600 pulses. Every stimulation set includes three sessions. All participants enrolled in the cTBS group will receive one stimulation set per day with an intersession interval (ISI) of more than $30 \mathrm{~min}$. Active treatment will be delivered over the right DLPFC at $80 \%$ of the resting motor threshold. The first stimulation set will be given at 4 hours after surgery on the day of surgery. The other two 
stimulation sets will be given at 8:00, 8:30 and 9:00 hours every day on postoperative day 1 and day 2 , or the day of discharge.

\section{Sham stimulation group}

The sham stimulation group will receive sham stimulation. Sham stimulation is designed with the same pattern (600 pulses per session, 3 sessions per set, ISI $\geq 30 \mathrm{~min}, 3$ sets). The only difference is that the coil is placed vertically $\left(90^{\circ}\right)$ to the skull for the sham stimulation, whereas the coil is placed centred over the right DLPFC for the actual stimulation. In addition, the stimulation time points will be the same as in the cTBS group; thus, participants will receive up to 5400 stimulation pulses (sham or active) during the study. To improve participants' adherence, we have contacted attending surgeons and anesthesiologists, and other staff to obtain their support for research. Moreover, we will provide detailed research-related information during recruiting, consultation, hospitalisation and follow-up.

\section{Study procedure and data collection \\ Participant screening and enrolment}

The participants will be screened on the day they are admitted to the hospital. Those participants who meet the inclusion criteria will be provided with informed consent on the same day. Then, the researchers will perform the assessments on the day before surgery (or Friday for participants who will undergo surgery the following Monday) using the methods described in our previous studies. ${ }^{101135}$ Participants who complete the preoperative assessments will be enrolled in the study.

\section{Preoperative assessments}

We will perform the preoperative assessment on the day before the surgery. The preoperative assessments will include the following:

- Demographic data: age, sex, education level and body mass index.

- Medical history and comorbid conditions, including the components of the Charlson Comorbidity Index.

- Main laboratory results (eg, blood routine and C reactive protein).

- Evaluation for incidence of delirium using CAM.

- Evaluation for cognitive function using MMSE, DSI Daily.

- Evaluation of ADL using ADL.

- Evaluation of pain using VAS.

- Evaluation of sleep using PSQI.

Anaesthesia, surgery and postoperative care

All participants will receive regular and routine care of anaesthesia, surgery and postoperative treatment, including postoperative analgesia. Meanwhile, all information on perioperative intervention to the participants will be recorded in detail. Relevant data will be used for post hoc analysis.
Postoperative assessments during hospitalisation

Participants will be evaluated twice daily up to 3 days following surgery or the time of discharge.

- Incidence and severity of delirium using CAM and CAM-S, respectively, during postoperative days 1-3.

- Cognitive function assessed using DSI Daily.

- ADL assessed using the ADL scale.

- Pain using VAS.

- Quality of sleep using PSQI.

- Length of hospital stay and discharge-to-facility or home.

- Complications and mortality.

Follow-up assessments

Participants will be evaluated 3 months after surgery.

- ADL assessed using the ADL scale.

- Pain using VAS.

- Cognitive function assessed using MMSE.

- Quality of sleep using PSQI and DSI Daily.

\section{Endpoint}

The endpoint is the completion of the final assessment at the follow-up. If the participant passes away for any reason before the endpoint, the time and cause of death will be recorded.

\section{Statistics and data management \\ Statistical analysis}

The researchers will wholly and accurately record the original research data using case report forms (CRFs). Data will be entered into the data set by two people, and the entered data will be compared and cross-checked to reduce the chances of mistakes. All statistical analyses will be processed using SPSS V.23.0 (SPSS). The Student's t-test and Mann-Whitney test will be used to determine differences between two groups of data with normal $($ mean $\pm \mathrm{SD})$ and non-normal distributions (median $\pm \mathrm{IQR}$ ), respectively. The $\chi^{2}$ test or Fisher's exact test will be used to compare the difference in categorical variables between groups. A significance level of $\mathrm{p}<0.05$ will be set for all tests. In addition, several subgroup analyses will be performed based on the following:

- Demographic data: age (youngest-old (from 65 to 74 years), middle-old (from 75 to 84 years) and oldest-old (>85 years $)),{ }^{36}$ education level.

- Anaesthesia: anaesthesia type and depth of anaesthesia.

- Surgery type and CCI.

Data will be reviewed for outliers and missing data. Then, multiple imputations will be performed to fill the missing data using Bayesian methods in SPSS. All analyses will be based on randomised participants.

\section{Quality control}

Before the study, the involved researchers will be trained to complete the study protocol and working plan and the CRFs. They will also be trained in considerations during the conduct of the study (eg, potential adverse events and complications). In addition, they will receive an introduction to TMS and guidelines for sham and active cTBS interventions. 
New training programmes will be organised according to the study progress. The DSMB, including independent and multidisciplinary experts (from the Clinical Research Office, Department of anesthesiology and Department of Psychiatry of the hospital), will recommend the continuation, modification or termination of the trial. ${ }^{37}$ The supervisor(s) will monitor the conduct of the study. To ensure integrity and adherence to the intervention of the cTBS and sham groups and the safety and efficacy of the data, the DSMB will closely monitor the intervention every 6 months after the intervention begins. This protocol is written in accordance with the Standard Protocol Items: Recommendations for Interventional Trials ${ }^{38}$ and Consolidated Standards of Reporting Trials guidelines. ${ }^{39}$ The trial protocol must be strictly adhered to throughout the trial period. All expected and unexpected findings will be documented to guarantee value reliability.

\section{Patient and public involvement}

Patients and the public were not involved in the study design and will not be involved in the study's recruitment, conduct or report. However, the study results will be disseminated to the public through public fora and seminars organised by the partnered agencies and the funder.

\section{Ethics and dissemination \\ Ethics Committee}

Ethical approval has been obtained from the ethics committee of Shanghai 10th People's Hospital (project number: SHSY-IEC-4.1/17-89/03, 3 January 2020, V.2.0). In addition, the principal investigator will submit research progress reports to the ethics committee regularly.

\section{Written informed consent}

Before obtaining consent, the researchers will ensure that the prospective participants adequately understand the purpose of the research study, the nature and extent of their participation, the potential benefits of the study, and the risks involved in a written informed manner. The researcher must inform every potential participant that they have the right to withdraw their consent at any time during the study period. The participant should initial each page of the consent form and sign the final page where indicated. The person obtaining consent must sign the form as well. The date of signature will also be recorded. The model informed consent has been included in online supplemental material. All written consent will be kept as a part of the clinical trial documents.

\section{Privacy protection}

The personal information of all participants will be kept confidential. We will assign each participant an identification (ID) number; thus, the data can be analysed by ID number. The ID numbers and patient linkage will be maintained in a secure (locked or passwordprotected) storage. Results of the study will be published in peer-reviewed journals without personal information (excluding name, age, sex, etc). The data and patient information from this study will be kept confidential and accessible to the study investigator.

\section{Safety considerations}

Currently, cTBS is considered to be a safe technique. Nevertheless, some studies suggest that a light headache and muscle cramps may occur during TMS or after treatment. ${ }^{40}$ In cases where contraindications and safety parameters are not noted, serious adverse events such as epilepsy may occur. ${ }^{41}$ The risk of cTBS, including epilepsy, is considered to be very low. ${ }^{42}$ To avoid adverse effects and risks to the participants, all procedures will follow the management security and application guidelines of cTBS during the study. ${ }^{41}{ }^{43}$ The potential complication of cTBS includes epilepsy, headache, dizziness, syncope and nausea. We will follow up with the participants up to 3 months after the anaesthesia/surgery. Participants will be questioned about adverse events, and this information will be recorded and published, including time of occurrence, diagnosis, time of diagnosis, management, duration of persistence and sequelae. If a severe adverse event (eg, seizure) occurs, the patient should be treated immediately and followed up until the problem completely resolves or when therapy is ended.

\section{DISCUSSION}

There are no clear guidelines for determining the most effective specific stimulation parameters, such as cTBS intensity or the number of total sessions and cumulated sessions per day. However, a recent study showed that stimulation with 1800 pulses per session in patients with refractory depression is safe and effective. ${ }^{44}$ Therefore, the proposed stimulation setting (600 pulses per session, 12 sessions over 4 days) is considered safe. Several potential practical and operational issues may pose challenges to the timely completion of this study, particularly regarding recruitment and attrition. We will use cTBS instead of rTMS in the proposed research because cTBS takes less time, reducing the participant drop-out rate. The effect of TMS is related to the cortical activation state. ${ }^{45}$ For example, most patients under remifentanilbased general anaesthesia attained complete orientation and return of pain sensation $30 \mathrm{~min}$ after anaesthesia and surgery. ${ }^{46}$ Therefore, the first set of stimulations, which will be performed at 4 hour after the end of the surgery, will not be influenced by the anaesthesia because the patients' sensation will have recovered before this time. ${ }^{46} 47$

This is a single-centre clinical trial for orthopaedic surgery. Further studies should consider a more extended follow-up period, more types of surgery and multicentre studies to assess the generalisability of the results. In summary, cTBS is a promising neuromodulatory technique that has shown benefit in improving cognitive function. We expect that the outcomes of this randomised controlled double-blind trial will provide clinical evidence regarding whether cTBS can decrease the incidence and severity of postoperative delirium. 


\section{TRIAL STATUS}

We have not started this trial at the time of submission of this manuscript.

\section{Author affiliations}

${ }^{1}$ Department of Psychiatry, Shanghai Tenth People's Hospital, Shanghai, China ${ }^{2}$ Anesthesia and Brain Research Institute, Tongji University School of Medicine, Shanghai, China

${ }^{3}$ Shanghai Mental Health Center, Shanghai Jiaotong University School of Medicine, Shanghai, China

${ }^{4}$ Geriatric Anesthesia Research Unit, Department of Anesthesia, Critical Care and Pain Medicine, Massachusetts General Hospital and Harvard Medical School, Charlestown, Massachusetts, USA

Acknowledgements We would like to express our gratitude to the Department of Orthopaedics in Shanghai 10th People's Hospital for their kind assistance. We are also thankful to Wuhan Yiruide Medical Equipment for facility support.

Contributors Study concept and design: YS, ZX and TY. Acquisition of data: XW, $\mathrm{DZ}, \mathrm{TT}, \mathrm{XM}, \mathrm{MW}$ and JS. Analysis and interpretation of data: XM, XW, TT, YS and ZX. Drafting of the manuscript: YS, ZX, XW and MW. Critical revision of the manuscript for important intellectual content: YS and ZX. Obtained funding: YS. Administrative, technical and material support: YS, ZX, DZ and TY. Study supervision: YS and ZX.

Funding This study will be supported by grant numbers 81720108012 and 81571034 from the National Natural Science Foundation of China (YS).

Competing interests None declared.

Patient consent for publication Not required.

Provenance and peer review Not commissioned; externally peer reviewed.

Supplemental material This content has been supplied by the author(s). It has not been vetted by BMJ Publishing Group Limited (BMJ) and may not have been peer-reviewed. Any opinions or recommendations discussed are solely those of the author(s) and are not endorsed by BMJ. BMJ disclaims all liability and responsibility arising from any reliance placed on the content. Where the content includes any translated material, BMJ does not warrant the accuracy and reliability of the translations (including but not limited to local regulations, clinical guidelines, terminology, drug names and drug dosages), and is not responsible for any error and/or omissions arising from translation and adaptation or otherwise.

Open access This is an open access article distributed in accordance with the Creative Commons Attribution Non Commercial (CC BY-NC 4.0) license, which permits others to distribute, remix, adapt, build upon this work non-commercially, and license their derivative works on different terms, provided the original work is properly cited, appropriate credit is given, any changes made indicated, and the use is non-commercial. See: http://creativecommons.org/licenses/by-nc/4.0/.

ORCID iD

Yuan Shen http://orcid.org/0000-0002-7811-1478

\section{REFERENCES}

1 Inouye SK, Westendorp RGJ, Saczynski JS. Delirium in elderly people. Lancet 2014;383:911-22.

2 Monk TG, Weldon BC, Garvan CW, et al. Predictors of cognitive dysfunction after major noncardiac surgery. Anesthesiology 2008;108:18-30.

3 Saczynski JS, Marcantonio ER, Quach L, et al. Cognitive trajectories after postoperative delirium. N Engl J Med 2012;367:30-9.

4 Pandharipande PP, Girard TD, Jackson JC, et al. Long-term cognitive impairment after critical illness. N Engl J Med 2013;369:1306-16.

5 Lundström M, Edlund A, Bucht G, et al. Dementia after delirium in patients with femoral neck fractures. J Am Geriatr Soc 2003:51:1002-6.

6 Fong TG, Jones RN, Shi P, et al. Delirium accelerates cognitive decline in Alzheimer disease. Neurology 2009;72:1570-5.

7 Marcantonio ER. Postoperative delirium: a 76-year-old woman with delirium following surgery. JAMA 2012;308:73-81.

8 Vutskits L, Xie Z. Lasting impact of general anaesthesia on the brain: mechanisms and relevance. Nat Rev Neurosci 2016;17:705-17.

9 Moskowitz EE, Overbey DM, Jones TS, et al. Post-operative delirium is associated with increased 5-year mortality. Am J Surg 2017;214:1036-8.
10 Shi Z, Wu Y, Li C, et al. Using the Chinese version of memorial delirium assessment scale to describe postoperative delirium after hip surgery. Front Aging Neurosci 2014;6:297.

11 Mei $\mathrm{X}$, Chen $\mathrm{Y}$, Zheng $\mathrm{H}$, et al. The reliability and validity of the Chinese version of confusion assessment method based scoring system for delirium severity (CAM-S). J Alzheimers Dis 2019;69:709-16.

12 Witlox J, Eurelings LSM, de Jonghe JFM, et al. Delirium in elderly patients and the risk of postdischarge mortality, institutionalization, and dementia: a meta-analysis. JAMA 2010;304:443-51.

13 AGS/NIA Delirium Conference Writing Group, Planning Committee and Faculty. The American geriatrics Society/National Institute on aging Bedside-to-Bench conference: research agenda on delirium in older adults. J Am Geriatr Soc 2015;63:843-52.

14 Fong TG, Davis D, Growdon ME, et al. The interface between delirium and dementia in elderly adults. Lancet Neurol 2015;14:823-32.

15 Leslie DL, Marcantonio ER, Zhang Y, et al. One-year health care costs associated with delirium in the elderly population. Arch Intern Med 2008;168:27-32.

16 Leslie DL, Inouye SK. The importance of delirium: economic and societal costs. J Am Geriatr Soc 2011;59 Suppl 2:S241-3.

17 Inouye SK, Bogardus ST, Baker DI, et al. The hospital elder life program: a model of care to prevent cognitive and functional decline in older hospitalized patients. Hospital elder life program. J Am Geriatr Soc 2000;48:1697-706.

18 Xu Y, Qiu Z, Zhu J, et al. The modulation effect of non-invasive brain stimulation on cognitive function in patients with mild cognitive impairment: a systematic review and meta-analysis of randomized controlled trials. BMC Neurosci 2019;20:2

19 Feng S-fang, Shi T-yao, Fan -Yang, et al. Long-Lasting effects of chronic rTMS to treat chronic rodent model of depression. Behav Brain Res 2012;232:245-51.

20 Thut G, Pascual-Leone A. A review of combined TMS-EEG studies to characterize lasting effects of repetitive TMS and assess their usefulness in cognitive and clinical neuroscience. Brain Topogr 2010;22:219-32.

21 Sandrini M, Umiltà C, Rusconi $\mathrm{E}$. The use of transcranial magnetic stimulation in cognitive neuroscience: a new synthesis of methodological issues. Neurosci Biobehav Rev 2011;35:516-36.

22 Chappell AJ. Cognitive performance as a function of right-sided, lowfrequency rTMS administration using CNS vital signs, 2016.

23 Lee SA, Kim M-K. Effect of low frequency repetitive transcranial magnetic stimulation on depression and cognition of patients with traumatic brain injury: a randomized controlled trial. Med Sci Monit 2018;24:8789-94.

24 Suppa A, Huang Y-Z, Funke K, et al. Ten years of theta burst stimulation in humans: established knowledge, unknowns and prospects. Brain Stimul 2016;9:323-35.

25 Cho SS, Ko JH, Pellecchia G, et al. Continuous theta burst stimulation of right dorsolateral prefrontal cortex induces changes in impulsivity level. Brain Stimul 2010;3:170-6.

26 Cho SS, Pellecchia G, Ko JH, et al. Effect of continuous theta burst stimulation of the right dorsolateral prefrontal cortex on cerebral blood flow changes during decision making. Brain Stimul 2012;5:116-23.

27 Ott DVM, Ullsperger M, Jocham G, et al. Continuous thetaburst stimulation (cTBS) over the lateral prefrontal cortex alters reinforcement learning bias. Neuroimage 2011;57:617-23.

28 Mensen A, Gorban C, Niklaus M, et al. The effects of theta-burst stimulation on sleep and vigilance in humans. Front Hum Neurosci 2014;8:420.

29 Annak O, Heidegger T, Walter C, et al. Effects of continuous theta-burst stimulation of the primary motor and secondary somatosensory areas on the central processing and the perception of trigeminal nociceptive input in healthy volunteers. Pain 2019;160:172-86

30 Inouye SK, Kosar CM, Tommet D, et al. The CAM-S: development and validation of a new scoring system for delirium severity in 2 cohorts. Ann Intern Med 2014;160:526-33.

31 Stern WM, Tormos JM, Press DZ, et al. Antidepressant effects of high and low frequency repetitive transcranial magnetic stimulation to the dorsolateral prefrontal cortex: a double-blind, randomized, placebocontrolled trial. J Neuropsychiatry Clin Neurosci 2007;19:179-86.

32 Rossini D, Lucca A, Magri L, et al. A symptom-specific analysis of the effect of high-frequency left or low-frequency right transcranial magnetic stimulation over the dorsolateral prefrontal cortex in major depression. Neuropsychobiology 2010;62:91-7.

33 Wu Y, Shi Z, Wang M, et al. Different MMSE score is associated with postoperative delirium in Young-Old and Old-Old adults. PLoS One 2015;10:e0139879. 
34 Albert MS, Levkoff SE, Reilly C, et al. The delirium symptom interview: an interview for the detection of delirium symptoms in hospitalized patients. J Geriatr Psychiatry Neurol 1992;5:14-21.

35 Shi Z, Mei X, Li C, et al. Postoperative delirium is associated with long-term decline in activities of daily living. Anesthesiology 2019;131:492-500.

36 Lee SB, Oh JH, Park JH, et al. Differences in youngest-old, middleold, and oldest-old patients who visit the emergency department. Clin Exp Emerg Med 2018;5:249-55.

37 Fleming TR, DeMets DL. Monitoring of clinical trials: issues and recommendations. Control Clin Trials 1993;14:183-97.

38 Chan A-W, Tetzlaff JM, Gøtzsche PC, et al. Spirit 2013 explanation and elaboration: guidance for protocols of clinical trials. BMJ 2013;346:e7586.

39 Moher D, Hopewell S, Schulz KF, et al. Consort 2010 explanation and elaboration: updated guidelines for reporting parallel group randomised trials. BMJ 2010;340:c869.

40 Gomes-Osman J, Field-Fote EC. Improvements in hand function in adults with chronic tetraplegia following a multiday $10-\mathrm{Hz}$ repetitive transcranial magnetic stimulation intervention combined with repetitive task practice. J Neurol Phys Ther 2015;39:23-30.

41 Rossi S, Hallett M, Rossini PM, et al. Safety, ethical considerations, and application guidelines for the use of transcranial magnetic stimulation in clinical practice and research. Clin Neurophysiol 2009;120:2008-39.

42 Oberman L, Edwards D, Eldaief M, et al. Safety of theta burst transcranial magnetic stimulation: a systematic review of the literature. J Clin Neurophysiol 2011;28:67-74.

43 Lefaucheur JP, Aleman A, Baeken C, et al. Evidence-Based guidelines on the therapeutic use of repetitive transcranial magnetic stimulation (rTMS): an update (2014-2018). Clin Neurophysiol 2020;131:1168-9.

44 Cole EJ, Stimpson KH, Bentzley BS, et al. Stanford accelerated intelligent neuromodulation therapy for treatment-resistant depression. Am J Psychiatry 2020;177:716-26.

45 Silvanto J, Pascual-Leone A. State-dependency of transcranial magnetic stimulation. Brain Topogr 2008;21:1-10.

46 Albrecht S, Fechner J, Geisslinger G, et al. Postoperative pain control following remifentanil-based anaesthesia for major abdominal surgery. Anaesthesia 2000;55:315-22.

47 Qi Y, Yao X, Zhang B, et al. Comparison of recovery effect for sufentanil and remifentanil anesthesia with $\mathrm{TCl}$ in laparoscopic radical resection during colorectal cancer. Oncol Lett 2016;11:3361-5. 